\title{
Sachkundenachweis und Hundeführerschein
}

\author{
Was Tierärzte wissen sollten
}

Celina del Amo

Mit den Begriffen Hundeführerschein oder Sachkundenachweis (für Hundehalter) werden landläufig Prüfungen benannt, die in verschiedenen Gesetzen bzw. Verordnungen aufgeführt werden, die zur Gefahrenabwehr durch das jeweilige Bundesland erlassen wurden. Aufgrund der landesrechtlich unterschiedlichen Regelungen zur Eindämmung der von Hunden ausgehenden potenziellen Gefahr ist in Deutschland diesbezüglich eine recht unübersichtliche Situation entstanden.

In manchen Bundesländern sind Prüfungen Pflicht, in denen nur theoretisches Wissen abgefragt wird, in anderen wiederum muss der Tierhalter auch praktisch unter Beweis stellen, dass er seinen Hund sicher und kontrolliert führen kann. Das hierbei jeweils erzielte Ergebnis wird je nach Gesetzeslage mitunter der generellen Sachkunde des Halters zugeschrieben oder gilt nur für das Zusammenspiel des Menschen mit dem konkret vorgestellten Hund.

Die Prüfungspflicht betrifft nicht immer die generelle Hundehaltung. In manchen Fällen beziehen sich die Regelungen nur auf die Haltung von Hunden bestimmter Rassen, Größen, Gewichtsklassen oder ab einem gewissen Alter der Tiere. Ähnliches gilt für spezielle Auflagen der Haltung (beispielsweise Leinen- oder Maulkorbpflicht), von denen der Hund mit bestandener Prüfung ggf. befreit werden kann.

\section{Gemeinsamkeiten aller Prüfungen}

Alle „Hunde-Gesetze“ und „Hunde-Verordnungen“ sind mit dem Ziel der Gefahrenabwehr inkl. des Schutzes unbeteiligter Dritter erlassen worden. Einer ver- antwortungsbewussten Art der Hundehaltung und -führung (speziell im öffentlichen Bereich) kommt somit eine Schlüsselrolle zu. Der Aspekt der Sicherheit und Rücksichtnahme wird in allen Prüfungen aufgegriffen. Darüber hinaus umfasst die Hundehaltung aber natürlich auch pflegerische, erzieherische und rechtliche Aspekte. Je nach Prüfung wird dem Hundehalter hier ebenfalls mehr oder weniger genau auf den Zahn gefühlt.

Für die mögliche Befreiung einer generell bestehenden Leinen- und Maulkorbpflicht sind die Inhalte der Prüfungen natürlich noch weiter gefasst. Hier stehen neben den Führungsqualitäten des Halters auch der Gehorsam und der Charakter des Hundes unmittelbar im Mittelpunkt. Konkret wird das Zusammenspiel des Hund-Halter-Teams, die Reaktionen des Hundes im Hinblick auf verschiedene Reizsituationen sowie dessen (prompte) Umsetzung bestimmter Gehorsamskommandos überprüft.

\section{Wichtige Hinweise für den Tierhalter}

\section{Unwissenheit schützt vor Strafe nicht}

Jeder Hundehalter ist grundsätzlich zur Einhaltung der jeweiligen bundes- und landesrechtlichen Bestimmungen verpflichtet, die für das Bundesland gelten, in dem er lebt bzw. sich mit seinem Hund gemeinsam aufhält. Unkenntnis im Hinblick auf die jeweils geltenden Gesetze/ Verordnungen schützt ihn daher - im Falle eines Verstoßes - nicht vor Strafe. Es ist daher sinnvoll, sich auch bei Reisen in andere Bundesländer vorab zu informieren, welche Regelungen für den eigenen Hund dort gelten.
Ist die Prüfung offiziell anerkannt?

Die Prüfungs- bzw. Trainingsinhalte hinter den Begriffen „Hundeführerschein“ bzw. „Sachkundenachweis“ spiegeln mitunter auch nur hundeschul-, club- oder vereinsinterne Regeln wider. Dies kann zu Verwirrung und Missverständnissen führen. Vor der Belegung eines Kurses in einer Hundeschule bzw. einem Hundeverein sollte daher geklärt werden, ob die angesetzte Abschlussprüfung behördlich anerkannt ist. Schließlich hat nur in diesem Fall ein möglicherweise erteiltes Zertifikat auch offiziell Gültigkeit.

\section{Prüfungsvorbereitung}

Gleichwohl welcher Art die Prüfung ist, die der Tierhalter möglicherweise ablegen muss, ist es grundsätzlich ratsam, sich vor dem Prüfungstermin eingehend mit den Inhalten zu beschäftigen. Werden auch praktische Aspekte (Führung und Gehorsam) beurteilt, lohnt es sich zudem, auch den Hund mit den Anforderungen vertraut zu machen und erzieherisch auf die Prüfung vorzubereiten. Dies erfolgt im Idealfall durch ein kleinschrittiges und belohnungsbasiertes Training. Literaturempfehlungen sowohl für die theoretischen als auch die praktischen Prüfungen sind am Ende des Artikels (unter Literatur) aufgeführt.

\section{Tierärztliche Aufgaben}

In der Kleintiersprechstunde hat der Tierarzt immer wieder Berührungspunkte mit den jeweils für die Hundehalter geltenden rechtlichen Anforderungen ihrer Tiere. So sollten die Tierhalter beim Besuch in der Praxis beispielsweise im Hinblick auf die ggf. erforderliche Kennzeichnungspflicht aufgeklärt werden. Im Bedarfsfall sollte auch die Kennzeichnung inkl. entsprechender Dokumentation im EU-Heimtierausweis vorgenommen werden. 


\section{Chip-Ablese-Training}

\section{Lernschritt}

Der Halter bewegt seine rechte Hand von vorne kommend an der linken Halsseite des Hundes auf und ab und gibt dem Hund nachfolgend mit der linken Hand ein Leckerchen, sodass der Hund seine Schnauze nach rechts richtet. Auf diese Weise wird nicht nur eine positive Erwartungshaltung kreiert und der Hund für seine „Geduld“ bei der Manipulation belohnt. Gleichzeitig wird auch ein Abwenden vom Untersuchenden geübt. Dies zielt darauf ab, den Hund auch auf Situationen vorzubereiten, in denen der Chip später einmal von einer fremden Person abgelesen wird.

\section{Lernschritt}

Der Halter wiederholt die gleiche Handlung, hält nun jedoch ein Handy oder ein anderes Gerät in der Hand, da dies von manchen Hunden als „bedrohlich“ empfunden wird. Es ist ok, den Hund das Gerät vorab begutachten zu lassen, jedoch ohne es ihm „aufzudrängen“.

\section{Lernschritt}

Der Hund kann nun mit einem Piepton vertraut gemacht werden, den viele Chiplesegeräte erzeugen, sobald sie den Chip ausgelesen haben. Dies kann beim Einsatz eines Handys beispielsweise durch die Aktivierung der Weckfunktion erfolgen. Bei Hunden, die geräuschängstlich sind, sollten in diesem Trainingsschritt besonders hochwertige Belohnungsleckerchen zum Einsatz kommen, um einen positiven Bezug zur Übung/Manipulation erzeugen zu können.

Auch das Ausstellen von Bescheinigungen ist eventuell erforderlich, wenn ein anderer Hund des Tierhalters möglicherweise bereits im Zeitraum XY in der Praxis betreut wurde. Je nach Gesetzeslage muss der Tierhalter seine Sachkunde bei einer durchgehenden Hundehaltung (bzw. der Hundehaltung mit einer maximalen Unterbrechung von 3 Monaten) nämlich nicht erneut unter Beweis stellen.

Ein weiterer optionaler Service in der Kleintierpraxis betrifft ebenfalls nur bestimmte Bundesländer. Die theoretische Sachkunde des Halters kann - bei entsprechendem Angebot der erforderlichen Prüfung - auch durch einen Tierarzt festgestellt werden. Auskunft darüber, welche Kriterien erfüllt sein müssen, um diesen Service anbieten zu können, erteilt die jeweilige Tierärztekammer.

Für alle Hundehalter gleichermaßen wichtig ist natürlich auch die umfassende klinische Betreuung des Hundes inkl. prophylaktischer Beratungen und der umgehenden Behandlung im Krankheitsfall. Die Erfahrung zeigt, dass zudem noch massiver Aufklärungsbedarf besteht, wie stark der Einfluss des körperlichen Wohlbefindens des Hundes auf dessen Verhaltensentwicklung und emotionale Steue- 
rung ist. Speziell, wenn das Zusammenspiel des Teams oder das Naturell des Hundes in einer praktischen Prüfung oder einem Wesenstest beurteilt wird, gewinnt dieser Aspekt noch einmal an Bedeutung.

\section{.konkret}

Das körperliche Wohlbefinden des Hundes hat stets direkten Einfluss auf seine Leistungsbereitschaft und Reaktionen in Reizsituationen.

Unterstützend können den Tierhaltern aber auch weitere allgemeine Informationen im Hinblick auf die Haltung und Führung des Hundes mit auf den Weg gegeben werden. Hierzu zählen Hinweise auf eine optionale Registrierung (beispielsweise bei TASSO) genauso wie die passive Unterstützung eines vorausschauenden Führungsstils (im Sinne der Aktion „Gelber Hund“, s. unten). Derartige Hilfestellungen können ohne besonderen Aufwand durch das Auslegen von Informationsmaterial in der Praxis erfolgen. Auch spezielle Literaturempfehlungen oder der Verkauf prüfungsrelevanter Hilfsmaterialien - z.B. Bücher und/oder Führungshilfsmittel - bieten sich an.

\section{"Gelber Hund“}

Das in Schweden ins Leben gerufene Projekt findet international immer mehr Anhänger. Es kommt Hunden und indirekt auch deren Haltern zugute, die aus irgendeinem Grund - sei es gesundheitlich oder charakterlich - mehr Freiraum bzw. Abstand zu Personen oder Artgenossen brauchen. Die betroffenen Hunde werden mit einem gelben Band oder Tuch an der Leine, dem Geschirr oder Halsband gekennzeichnet. Aus Gründen der freundlichen Rücksichtnahme sollte in Begegnungen mit diesen Tieren diskussionslos auf die Einhaltung des gewünschten $\mathrm{Ab}$ stands geachtet werden. Weitere Infos inkl. des kostenlosen Downloads für Plakate und Informationsmaterial sind unter www.gulahund.de zu finden.

\section{Vorbereitung ist die halbe Miete}

\section{Maulkorbtraining}

Unabhängig von einer aus rechtlichen Gründen möglicherweise bestehenden Maulkorbpflicht, stellt das frühzeitige Vertrautmachen mit dem Tragen eines Maulkorbs für jeden Hund eine sinnvolle Grundübung dar. In aller Regel ist ein wenig Überzeugungsarbeit nötig, um dem Halter das Maulkorbtraining „schmackhaft“ zu machen. Im Gespräch mit dem Tierhalter sollte daher neben der Freude, die der Hund bei einem kleinschrittigen Übungsaufbau unter zielgerichtetem Einsatz von Futterbelohnungen hat, auch der Aspekt der prophylaktischen Stressminimierung für den Hund deutlich herausgestellt werden. Hinweise, dass der Einsatz eines Maulkorbs die Abläufe bestimmter Praxissituationen erleichtern kann und er zudem unabhängig von der Rassezugehörigkeit des Hundes - auch bei Fahrten mit bestimmten öffentlichen Verkehrsmitteln im In- und Ausland vorgeschrieben ist, sollten an dieser Stelle nicht fehlen. Eine kostenlose schrittweise Trainingsanleitung, die dem Tierhalter an die Hand gegeben werden kann, ist unter www.vettext-manufaktur.de zu finden.

\section{Ablesen des Mikrochips}

Viele Hunde reagieren mit Scheu auf das Ablesen des Mikrochips. Sie fühlen sich vor allem durch die für das Ablesen erforderliche Nähe und Konzentration des Untersuchenden sowie durch das Gerät selbst oder die mit ihm umgesetzten Bewegungen bedroht. In Abhängigkeit des individuellen Stresslevels und erlernter Verhaltensstrategien des Hundes sind im Einzelfall auch aggressive Reaktionen beim Chip-Ablesen möglich. Zu beklagen ist, dass das Ablesen des Mikrochips nur in den seltensten Fällen in einen Trainingsplan aufgenommen wird. Dies liegt vermutlich daran, dass es so selten erfolgt bzw. erforderlich ist. Das ist schade, weil auch hier das bereits beim Maulkorbtraining Gesagte gilt: Die Übungen sind einfach umzusetzen, sie machen dem Hund Spaß und sind im Hinblick auf sein Stresserleben von hohem Wert $(\triangleright \mathbf{s}$. Kasten).

Speziell die Halter der Hunde, die eine Hundeführerscheinprüfung anstreben, sollten über die Möglichkeit eines Chip-Ablese-Trainings in Kenntnis gesetzt werden.

Hierbei spielt es keine Rolle, ob sie die Prüfung aus Pflichtgründen, zur Erreichung einer speziellen Befreiung von Leine oder Maulkorb oder im Rahmen eines privat angestrebten hundeschuloder clubinternen Hundeführerscheins anstreben. Letztlich zählt in allen Fällen die entspannte Vertrautheit des Hundes mit der Aktion.

Online zu finden unter

http://dx.doi.org/10.1055/s-0042-102847

\section{Literatur}

1 del Amo C. Sachkundenachweis für Hundehalter: So bestehen Sie den Hundeführerschein. 2. Aufl. Stuttgart: Eugen Ulmer; 2016

2 del Amo C, Jones-Baade R, Mahnke K. Der Hundeführerschein - Basiswissen und Fragenkatalog. 4. Aufl. Stuttgart: Eugen Ulmer; 2009

3 Metz G, Schalke E. Hundeführerschein und Sachkundenachweis - mit Frage-AntwortKatalog des VDH. Stuttgart: Franckh Kosmos; 2012

4 Sprauer S. Der Hundeführerschein - Lizenz zum Gassigehen. München: Bassermann; 2014

\section{Celina del Amo}

Lupologic - Zentrum für angewandte Kynologie und klinische Ethologie

Linienstraße 72

40227 Düsseldorf

info@lupologic.de 